\title{
ESTRUTURA E ORGANIZAÇÃO DO SISTEMA DE INFORMAÇÕES GEORREFERENCIADAS DE SOLOS DO BRASIL (SIGSOLOS - VERSÃO 1.0)(1)
}

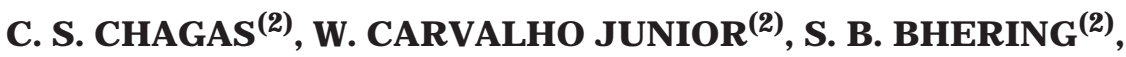 \\ A. K. TANAKA(3) $\&$ J . F. M. BACA ${ }^{(2)}$
}

\begin{abstract}
RESUMO
Os sistemas de informação armazenam informações detalhadas sobre o recurso terra, de modo que estas possam ser acessadas, combinadas e analisadas, sob vários pontos de vista, para os mais variados usos. No Brasil, a disponibilização das informações geradas nos levantamentos de solos tem sido pouco eficiente, em razão do grande volume, complexi dade e pouca normatização. Assim, com o objetivo de possibilitar o armazenamento, manipulação e disponibilização das informações sobre os solos brasileiros, foi desenvolvido o SigSolos. O SigSolos foi estruturado para permitir o armazenamento de informações oriundas de diferentes fontes, níveis e escalas de levantamentos de solos, tendo, como base, normas e conceitos utilizados na ciência do solo. A entidade "Trabalho" foi estabelecida como entidade central do sistema para reduzir a necessidade de compatibilizações e normatizações na aquisição dos dados, considerando as diferentes fontes e níveis de levantamento de solos existentes no Brasil. A 1a etapa do SigSolos foi concluída com a disponibilização da versão 1.0, para o armazenamento de dados, e, atualmente, já é possível acessar algumas informações sobre os solos brasileiros, a partir da Iniciativa Solos.br, diretamente na página da E mbrapa Solos na internet.
\end{abstract}

Termos de indexação: sistema de informação, SIG, solos, banco de dados

(1) Recebido para publicação em julho de 2003 e aprovado em agosto de 2004.

(2) Pesquisador Embrapa Solos. Rua J ardim Botânico 1024. CEP 22460-000 Rio de J aneiro (RJ ). E-mail: cesar; waldir; silvio; jesus\}@cnps.embrapa.br

(3) Professor do Instituto Militar de Engenharia - IME. Pça Gal. Tibúrcio 80. CEP 22290-270 Rio de J aneiro (RJ ). E-mail: tanaka@ime.eb.br 


\title{
SUMMARY: ORGANIZATION AND STRUCTURE OF THE BRAZILIAN SOIL INFORMATION SYSTEM (SIGSOLOS - VERSION 1.0)
}

\begin{abstract}
Information Systems store detailed information on land resources, so that thesecan be accessed, combined, and analyzed for several purposes from different points of view. In Brazil, the information produced in soil surveys has not been efficiently made available, due to the great volume, compl exity, and littlestandardization. Thus, with the objective of enabling the storage, manipulation, and disposal of information on Brazilian soils, the SigSol os System was devel oped. SigSol os was structured to all ow thestorage of information originated from different sources, levels, and scales of soil surveys, on well-known underlying soil concepts. Theentity "Work" was established as thecentral entity of thesystem, reducing the need for compatibilization and standardizations in the data acquisition, due to the different sources and levels of Brazilian soil surveys. The first stage of SigSolos was concluded, and version 1.0 is now availablefor data storage. By now, someinformation on B razilian soi Is can al ready beaccessed at I niciativa Sol os.br, di rectly on theinternet website Embrapa Solos.
\end{abstract}

Index terms: information systems, GIS, soils, database.

\section{NTRODUÇÃO}

Nas úl timas décadas, a rápi da evol ução dos métodos einstrumentos para aquisição, armazenamento, recuperação, manipulação, análises, acessibilidade e distribuição de dados tem facilitado enormemente o tratamento, pelos cientistas de solos, de grandes quantidades de dados, indispensáveis ao monitoramento eavaliação do sistema terra, de seus componentes e processos (Baumgardner, 1999).

O Brasil ocupa uma posição de destaque na real ização de estudos de sol os tropicais, tendo gerado grande quantidade de dados por meio de levantamentos de sol os eoutros estudos. No entanto, a disponibilização destas informações tem-se mostrado pouco eficiente, em razão do grande volume, complexidade e pouca normatização dos dados, o quetem dificultado elimitado sua utilização pel os usuários (Embrapa, 1998).

Segundovan Engelen (1999), os mapas tradicionais esuas versões digitalizadas, independentemente de suas escalas, não fornecem toda a informação requerida pelos usuários, já que a maioria dos dados coletados durante os levantamentos de solos não pode ser mostrada nos mapas ou não consta nas legendas. Assim, para facilitar a disponibilização da informação, são utilizados sistemas automatizados que possibilitam a entrada, armazenamento, processamentoesaída destas informações, permitindo aos usuários explorarem, de maneira mais rápida e segura, as informações requeridas para os diversos fins.

Os sistemas de informação de solos são relativamente recentes e buscam descrever os solos em seu ambiente natural. Estes podem ser descritivos e apresentar dados quantitativos com dimensões espaciais (unidades de mapas), ou dados pontuais, que descrevem equantificam propriedades específicas de um perfil de solo em determinado local etempo na superfície da terra (Baumgardner, 1999).

Diante da necessidade de informações para o desenvolvimento de estudos que envolvem a local ização, qualidade equantificação de propriedades de solos, seus processos esuas interações com outros recursos naturais (Ernstrom \& Lytle, 1993), organismos internacionais e países têm desenvol vido sistemas de informações de solos. Dentre estes, destacam-se: o Mapa Digital de Solos do Mundo (FAO, 1996), OSOTER - The World Soils and Terrain Database (van Engelen \& Wen, 1995), O CANSIS Canadian Soil Information System (MacDonald \& Kloosterman, 1984) e o NASIS - National Soil Information System (Soil Survey Staff, 1991).

O mapa de solos do mundo da FAO, na escala de 1:5.000.000, éo único banco gl obal de dados desolos existente. Ao longo dos anos $80 \mathrm{~s}$ e $90 \mathrm{~s}$, muitas tentativas foram feitas para digitalizar este mapa, existindo, atualmente, disponíveis para consulta várias versões em diferentes formatos (vetor eraster). Este mapa contém informações sobre a composição das unidades de mapeamento, em termos de tipo de solo, textura da camada superficial e classe de declividade do solo dominante, além da eventual presença de fases, tais como: caráter salino, sódico, petrocálcico, dentre outros (Nachtergaele, 1999).

O projeto SOTER nasceu da necessidade de atualização e expansão do banco de dados de solos usado pelo Mapa de Solos do mundo da FAO e representa o primeiro esforço para compilar um conjunto de dados globais na escala de 1:1.000.000. O conceito geral doSOTE R sebaseia no mapeamento de áreas de terra (unidades SOTER), as quais se apresentam distintas quanto aos padrões da paisagem, litologia, forma da superfície, declividade, 
material de origem e solos. Embora tenha sido inicialmente desenhado para o uso na escala de 1:1.000.000, o método é aplicável a escalas maiores junto com o desenvolvimento de bancos de dados nacionais. Um primeiro teste já foi realizado com os dados do mapa de solos do estado de São Paulo na escala de 1:500.000. Dentre as aplicações do SOTER está a possibilidade deavaliação do risco de erosão, potencial agrícola das terras, condição de salinidade e degradação das terras (van Engelen, 1999).

O Sistema de Informação de Solos do Canadá (CANSIS), desde 1972, tem dado suporte às atividades da agência de pesquisa canadense (Agriculture and Agri-F ood Canada - AAFC). O CANSIS foi desenvolvido para manipular dados de solo, clima, uso da terra, rendimento das culturas e dados geográficos, sendo os dados de solos parte integrante do National Soil Database (NSDB), cujo sistema eprocedimentos deSI G foram implementados no aplicativo Arc/I nfo (ESRI,1994).

O NSDB é um conjunto de arquivos que contém dados de solo, paisagem eclima para todas as regiões do Canadá. As informações sobre as características e distribuição dos solos são apresentadas em três níveis de detalhe: o mapa de solos na escala de 1:5.000.000; as paisagens de solos (SLC) na escala de 1:1.000.000; elevantamentos de solos detal hados, em escalas quevariam de 1:20.000 a 1:250.000 (Coote \& MacDonald, 1999).

O Departamento de Agricultura dos Estados Unidos, pel os levantamentos desol os, tem fornecido, desde 1886, informações de sol os para um número variado de usuários. No fim dos anos 60 s e início dos anos 70s, os cientistas da National Cooperative Soil Survey (NCSS) reconheceram a necessidade e o potencial de automação da grande quantidade de informações geradas nos levantamentos de sol os que estavam sendo executados. Desta maneira, foi desenvolvido o National Soil I nformation System (NASIS), para facilitar e melhorar a aquisição, manejo e distribuição destas informações. Semelhantemente ao sistema canadense, o NCSS desenvolve e mantém seis conjuntos de dados, que são o registro de caracterização de solos (SCR), registro de unidades de mapeamento (MUR), registro das unidades taxonômicas (TUR), banco de dados geográficos de levantamento de solos (SSURGO), banco de dados geográficos estadual (STASTGO) e banco de dados geográficos nacional (NATSGO). Cada um destes conjuntos foi desenvolvido para uma proposta ou necessidade específica (Lytle, 1993; 1999).

Cabe ressaltar que nenhum dos sistemas mencionados utiliza gerenciadores de banco de dados estendidos, capazes detratar dados espaciais. Desta forma, todos utilizam um aplicativo de sistema de informação geográfica, predominantemente segundo o model o vetorial, para o armazenamento dos dados geométricos (localização e extensão de objetos representados por pontos, linhas ou superfícies, e topologia) e para o armazenamento dos dados nãoespaciais, há um predomínio do emprego de aplicativos de sistemas de gerenciamento de banco relacional de dados comercialmente disponíveis (van Engelen \& Wen, 1995; MacDonald \& Kloosterman, 1984; Soil Survey Staff, 1991).

Estetrabalho objetivou apresentar aos cientistas de solos do Brasil e demais usuários da informação de solos a estrutura e organização do Sistema de Informações Georreferenciadas de Solos do Brasil (SigSolos - versão 1.0), que foi desenvolvido pela Embrapa Solos (E mbrapa, 1998), com a finalidade de permitir uma melhor organização, sistematização e gerenciamento da informação de solos do País.

\section{MATERIAL E MÉTODOS}

No desenvolvimento do projeto do SigSolos, foi utilizada, como estratégia, a opção por uma arquitetura dual, que consiste na interação de um sistema gerenciador de banco de dados (SGBD) relacional-objeto com um sistema de informação geográfica (SIG) (Figura 1), já que os SGBDs não dispõem dos tipos de dados e operadores apropriados para objetos geográficos e os SIGs não têm a capacidade de gerenciar eficazmente grandes volumes de dados não-espaciais. Assim, os usuários têm acesso ao banco de dados dir etamente por meio do SGBD ou da interface geográfica fornecida pelo SIG. A entrada de dados é feita por meio de um subsistema com interface visual baseada em formulários e menus. A abordagem empregada, por ser independente de aplicativo, permitea utilização de diferentes SGBDs e SI Gs (Tanaka et al., 1997).

Os bancos de dados relacionais armazenam dados em tabelas (relações matemáticas) interligadas por campos-chave. O modelo de dados é mais bem entendido em um nível de abstração mais el evado do que o das tabelas, por meio de um esquema conceitual representando entidades, que podem ser entendidas como al go da realidade modelada em que se deseja manter informações no banco de dados, podendo representar tanto objetos concretos quanto

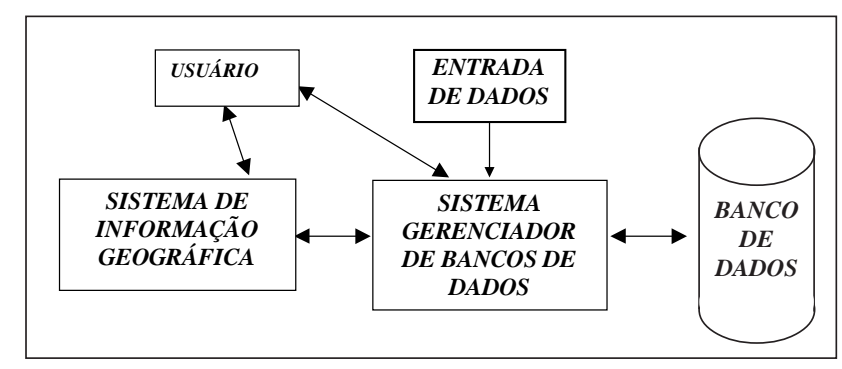

Figura 1. Arquitetura de software do SigSolos. 
objetos abstratos; relacionamentos, que são conjuntos de associações entre entidades; eatributos, que são definidos como características relevantes associadas a cada ocorrência de entidade ou relacionamento (Chen, 1976).

O modelo conceitual do SigSolos (Figura 2) foi definido a partir de consultas a especialistas em ciência do solo, com vistas em obter um melhor entendimento das entidades envolvidas e seus relacionamentos. Posteriormente, este foi implementado no aplicativo de desenho de banco de dados ERWin que, por ser independente de SGBD, permite criar diversas versões na maioria dos SGBDs existentes no mercado (Tanaka et al., 1996). O esquema resultante da modelagem do SigSol os é semelhante ao dos modelos encontrados na literatura internacional (Fernández \& Rusinkiewicz, 1993), com as devidas adaptações decorrentes das peculiaridades do sistema de classificação de solos adotado no Brasil.

O esquema conceitual foi desenvolvido em um modelo de representação chamado de IDEFIX, no qual aparecem apenas as entidades mais significativas e seus relacionamentos. O model o detal hado contém também entidades associativas, além de dezenas de tabelas de valores de domínios para atributos. Apenas os atributos-chave das entidades são mostrados, sendo os demais armazenados em dicionário de dados e não exibidos na figura para facilitar a visual ização do esquema (al gumas entidades possuem dezenas de atributos). Na figura 2, os componentes do model o espacial são representados em cinza-claro, enquanto as entidades unidade de mapeamento e ponto de amostragem, delineadas em cinza-escuro, são os el os de ligação entre o banco de dados descritivos e o banco de dados geográfico.

$\mathrm{Na}$ fase de estruturação do sistema, foi feita a validação através de protótipo de entrada de dados em linguagem SQL Windows, usando o SGBD Openingres (Computer Association, 1995) e o aplicativo de SIG ArcView (ESRI, 1996). No atual estádio de desenvolvimento, a versão monousuária do banco de dados está implementada no SGBD Microsoft Access, com o subsistema de entrada de dados em Access Basic e a visualização geográfica em ArcView (ESRI, 1996).

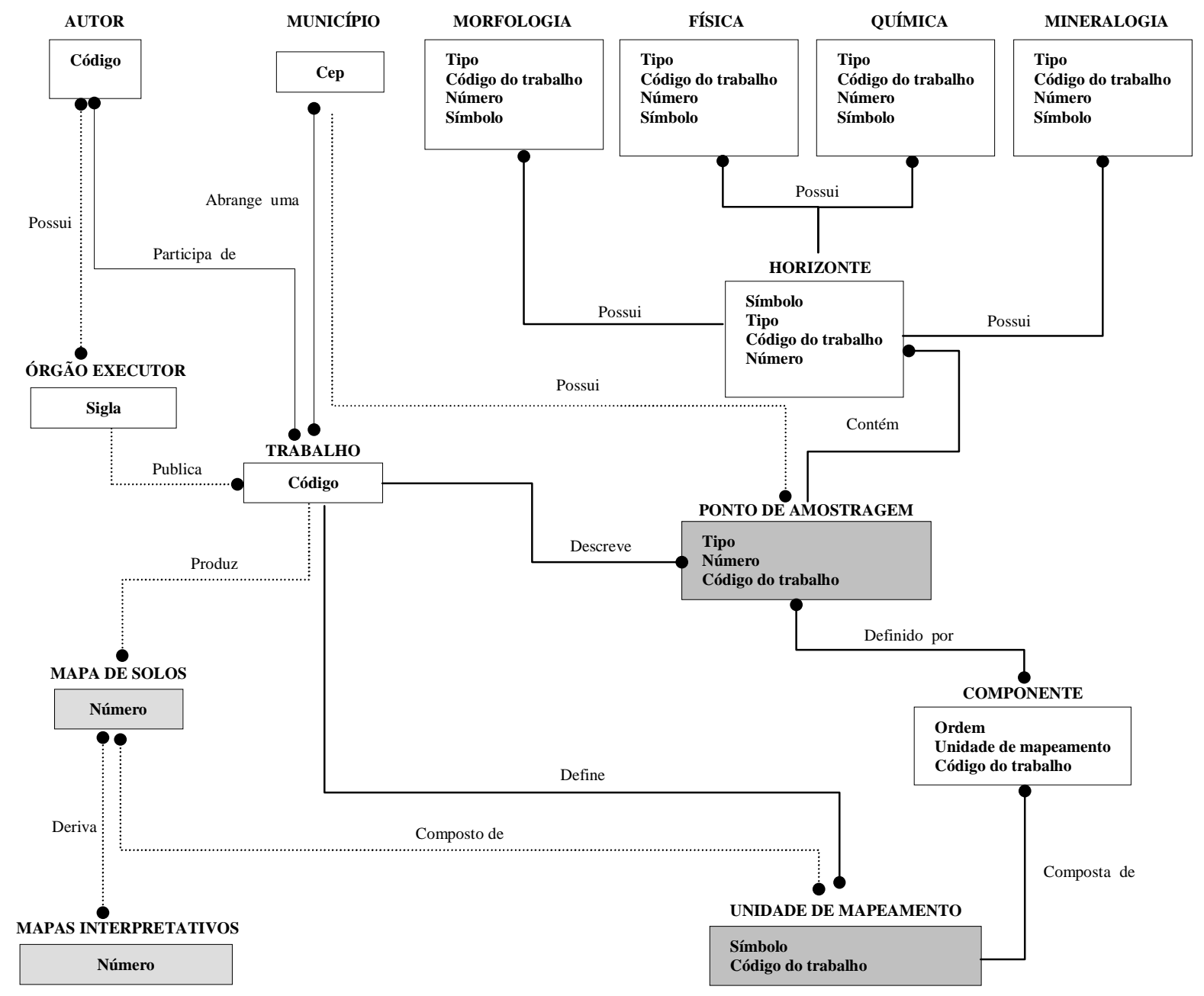

Figura 2. Modelo conceitual simplificado do SigSolos. 
A elaboração do SigSolos vem consolidar a necessidade de formalização dos conceitos básicos empregados na ciência do solo. Assim, os procedimentos empregados na organização dos dados apresentam uma visão sistematizada e estão baseados em normas e conceitos contidos em publicações nacionais (Embrapa, 1988a,b; Curi et al., 1993; E mbrapa, 1995; Ramalho Filho \& Beek, 1995; Lemos \& Santos, 1996; E mbrapa, 1997, 1999).

A base de informações foi desenhada para permitir o armazenamento de informações de sol os oriundas de diferentes fontes de dados, tais como: levantamentos de solos, teses e artigos científicos. Diferentemente dos sistemas de informação de solos canadense e americano, o SigSolos foi estruturado para armazenar informações de levantamentos de solos de diferentes níveis e escalas. Os dados no sistema foram cuidadosamente separados por campos independentes, para facilitar a recuperação das informações de forma isolada ou mesmo combinada.

A definição do "Trabal ho" como entidade central do sistema teve a finalidade de reduzir, ao máximo, a necessidade de compatibilizações enormal izações no processo de aquisição dos dados, mediante as diferentes fontes de dados e níveis de levantamento de solos existentes no Brasil. A inexistência de vínculo entre os diferentes estudos é uma característica do sistema que visa torná-lo ágil na recuperação e tratamento dos dados.

Após a definição do modelo conceitual, foi estabelecido que o sistema de entrada de dados apresentaria os seguintes requisitos: cadastros de institui ções eautores, dos trabal hos real izados pel os diferentes órgãos executores, das metodologias de determinações utilizadas nos trabal hos, dos pontos de amostragem, dos horizontes, dos resultados das determinações físicas, químicas e mineralógicas dos horizontes, das unidades de mapeamento e dos componentes; relatórios dos pontos de amostragem por estados e municípios e dos trabal hos por estado; navegação al eatória nos diferentes registros de uma tela (tabela) e edição nas tel as: inserção, atual ização, consulta e exclusão de registros da tabela associada à tela.

A seguir, foram projetadas as interfaces deentrada de dados, considerando que deveriam ter um mapeamento direto com a estrutura do banco de dados. Assim, buscou-se um refinamento contínuo e interativo entre o model o conceitual e o modelo funcional. As telas queformama interface deentrada de dados doSigSol os têm uma estrutura hierárquica que pode ser resumida na figura 3. Este modelo funcional foi implementado no aplicativo Microsoft Access 2.0 pela facilidade de emprego destesoftware.

As diferentes telas que formam a interface do sistema foram cuidadosamente elaboradas de tal maneira que a navegação refletisse as atividades realizadas durante o trabalho, de maneira convencional. Tal sistema, aliado às facilidades do Microsoft Access 2.0, permite que seu uso seja intuitivo e simplificado, sem trazer ao usuário mudanças bruscas na maneira de desenvolver suas atividades.

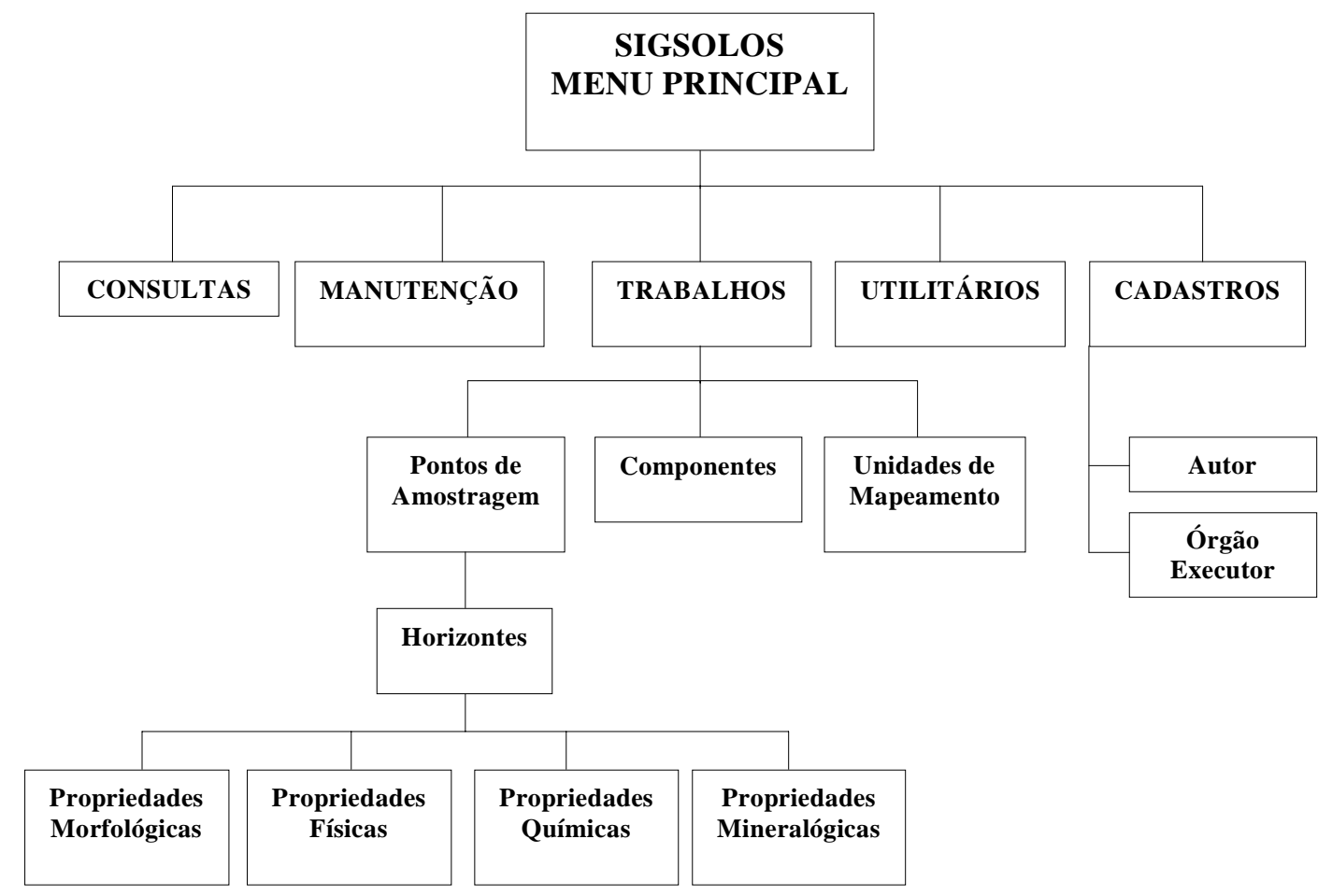

Figura 3. Estrutura hierárquica da funcionalidade da entrada de dados do SigSolos. 


\section{RESULTADOS}

Para atender às exigências e demandas específicas dos usuários das informações de solos, o sistema é formado por subsistemas, vinculados à entidade central "Trabalho". No primeiro subsistema, a entidade der eferência éo "Ponto de amostragem" (PA), deexistência real edomínio espacial pontual, caracterizada por diversas propriedades. O Ponto deamostragem tem no seu georreferenciamento um atributo importante de integração dos dados tabulares com as respostas espaciais. Em um nível hierárquico inferior, destaca-se a entidade "H orizonte" que está subdividida em morfologia, física, química e mineralogia, conforme já apresentado no modelo conceitual (Figura 2). O segundo subsistema está relacionado com os mapas gerados nos levantamentos de solos, visto que descreve as propriedades alfanuméricas da entidade "Unidadedemapeamento" (UM). Na caracterização desta entidade, é imprescindível a utilização do conceito de componente, outra entidade do sistema, pois nesta residem as propriedades rel evantes das uni dades de mapeamento.

A ligação entre os dois subsistemas pode ser feita por três caminhos distintos: pela localização, por meio de operadores espaciais; pela entidade Trabalho, ou por um campo definido na entidade Ponto de amostragem, denominado "componente definido", onde se pode ou não vincular um componente a determinada instância do Ponto de amostragem.

O SigSolos pode armazenar dados de vários tipos de estudos existentes. Neste sentido, os levan- tamentos de sol os podem ser consi derados como estudos completos, pois abrangem todo o conjunto de dados do sistema e os relacionamentos entre horizontes e suas propriedades, pontos de amostragem, componentes e unidades de mapeamento, ou aspectos espaciais. Outros tipos de estudos que oSigSol os pode comportar são os trabal hos técnico-científicos (artigos e teses, principalmente) que, usualmente, tratam apenas com a entidade ponto deamostragem, bem como os estudos de interpretação dos mapas de solos, como aptidão e zoneamento agrícolas, que usam dados de pontos descritos em levantamentos de solos.

No SigSolos, os pontos de amostragem e as unidades de mapeamento são os el os de ligação entre o banco de dados e o SIG. Por meio da interface geográfica do SIG, é possível visualizar os pontos e as áreas descritos no banco de dados, pela conexão cliente-servidor entreSI G eSGBD, e de consulta ao banco de dados usando a linguagem de mani pulação de dados do SGBD. U ma vez visualizados os dados, podem ser realizadas as operações espaciais disponíveis no SIG, como interseção, união, pertinência, proximidade e reclassificação, que, certamente, serão de grande utilidade na maioria das aplicações previstas no projeto (Tanaka et al., 1997).

O modelo conceitual detal hado do sistema de informações de solos contém também entidades associativas, assim como dezenas de tabelas de valores de domínios para atributos. No quadro 1, encontra-se uma listagem das principais entidades do sistema e seus respectivos dados.

\section{Quadro 1. Listagem dos principais elementos das entidades do SigSolos}

\begin{tabular}{|c|c|c|c|}
\hline Entidade & $\begin{array}{l}\text { Entidade } \\
\text { associativa }\end{array}$ & Dado mais relevante & Descrição \\
\hline \multirow[t]{3}{*}{ Trabalho } & Dados Gerais & $\begin{array}{l}\text { Título } \\
\text { Ano de publicação } \\
\text { Tipo de publicação } \\
\text { Nível do levantamento } \\
\text { Órgão executor } \\
\text { Laboratório responsável } \\
\text { Escala de publicação }\end{array}$ & $\begin{array}{l}\text { Título do trabal ho publicado } \\
\text { Ano de sua publicação } \\
\text { Tipo de publicação do trabalho } \\
\text { Nível do levantamento de sol os utilizado } \\
\text { Órgão responsável pela execução do trabalho } \\
\text { Laboratório } \\
\text { Escala utilizada na confecção do mapa de solos }\end{array}$ \\
\hline & Metodologia & $\begin{array}{l}\text { Método de prospecção } \\
\text { Material básico }\end{array}$ & $\begin{array}{l}\text { Metodologia utilizada no levantamento de solos } \\
\text { Tipo de material básico utilizado no levantamento de solos }\end{array}$ \\
\hline & $\begin{array}{l}\text { Métodos } \\
\text { físicos, } \\
\text { químicos e } \\
\text { mineralógicos }\end{array}$ & Vários & $\begin{array}{l}\text { Apresenta a referência bibliográfica dos métodos } \\
\text { analíticos empregados no trabalho }\end{array}$ \\
\hline $\begin{array}{c}\text { Unidade de } \\
\text { Mapeamento } \\
\text { (UM) }\end{array}$ & Dados Gerais & $\begin{array}{l}\text { Título do trabalho } \\
\text { Símbolo } \\
\text { Tipo } \\
\text { Área } \\
\text { Componentes } \\
\text { Inclusões e, ou, variações }\end{array}$ & $\begin{array}{l}\text { Título do trabalho ao qual a UM está vinculada } \\
\text { Símbolo da unidade de mapeamento } \\
\text { Tipo da unidade de mapeamento } \\
\text { Área ocupada pela UM no trabalho } \\
\text { Componentes relacionados com a UM } \\
\text { Inclusões e, ou, variações encontradas na UM }\end{array}$ \\
\hline
\end{tabular}




\section{Quadro 1. Continuação}

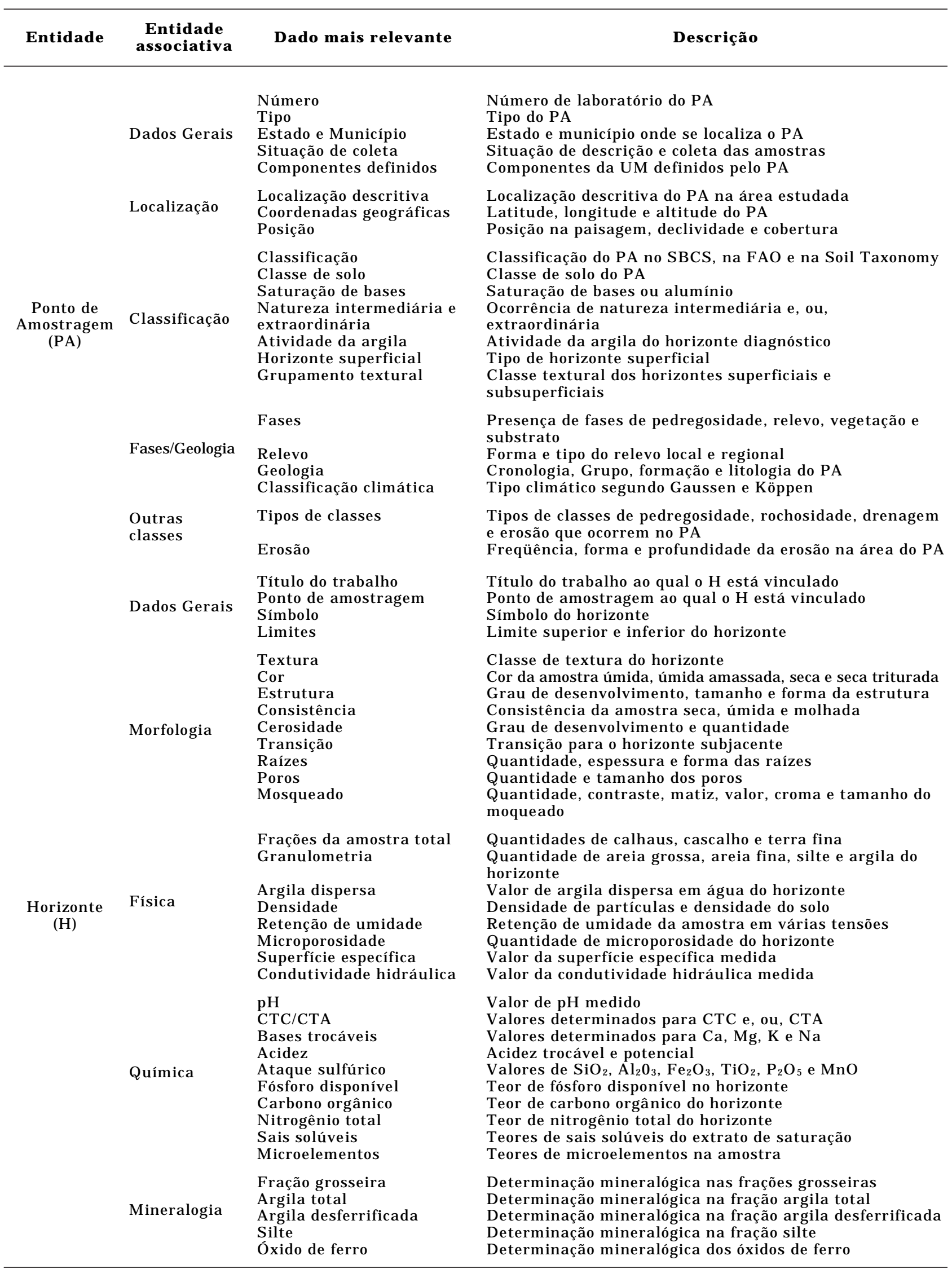


Segundo Ernstrom \& Lytle (1993), o componente de uma unidade de mapeamento é a entidade mais elementar, relativamente mais homogênea e identificável de uma área, para a qual uma série de val ores de propriedades podeser armazenada. Esta abrange uma extensão espacial e dispõe de um conjunto de propriedades que define suas características. De acordo com o Soil Survey Staff (1999), o pedon é mais elementar do que o componente; entretanto, este é amostrado em um local usando um ou mais perfis e, conseqüentemente, tem apenas um valor para cada propriedade descrita. Desta forma, para formar um componente, são normal mente necessárias combi nações de val ores de dois ou mais pedons.

No SigSolos, a entidade "Componente" foi introduzida no sistema para possibilitar o mapeamento entre as observações de campo e as legendas dos mapas de solos, em virtude da falta de relacionamento, em al guns levantamentos desolos, denível generalizado eatémesmo de reconhecimento, entre o(s) componente(s) de uma(s) unidade(s) de mapeamento e ponto(s) de amostragem, ou seja, componentes que são definidos sem quetenham sido feitos um exame eanálise de um perfil. Assim, uma característica importante do SigSolos é a redundância controlada, ou seja, repetição proposital de informações entre pontos de amostragem e componente para as propriedades diagnósticas.

Concomitantemente com o desenvolvimento do modelo conceitual, procedeu-se à elaboração das tabelas de domínio de valores. Assim, o sistema conta com aproximadamente 80 tabelas de domínio, que foram estabelecidas com base nos conceitos e normas definidas e, ou, adotadas pela Sociedade Brasileira de Ciência do Solo (E mbrapa, 1998). A importância desta organização reside no fato de, além de diminuir o tamanho do banco de dados, servir de instrumento sistematizador das informações de sol os, evitando a utilização e prol ifer ação de termos não reconhecidos pela sociedade. No entanto, os administradores do sistema deverão estar atentos a todas as modificações e inovações que, porventura, sejam introduzi das oficialmente na Ciência do Solo, em virtude de sua constante evolução. Considerando o grande número de tabelas de domínios de val ores, serão apresentados apenas dois exemplos destas (Quadros 2 e 3).

O estabelecimento de tabelas de domínio de val ores implica a necessidade de atualização e, ou, normatização da terminol ogia e conceitos adotados ao longo de várias décadas de levantamentos desol os no Brasil, o que permitirá o tratamento dos dados de maneira mais abrangente e segura.

As tel as do aplicativo doSigSolos sãoas interfaces de entrada de dados no sistema. Os atributos que foram definidos nas diferentes telas correspondem aos atributos definidos nas tabelas de domínio do banco de dados. $\mathrm{Na}$ entrada dos dados, foram impostas

\section{Quadro 2. Tabela de domínio de valores referentes ao nível de levantamento de solos}

\begin{aligned} & \hline Código \multicolumn{1}{c}{ Descrição } \\ & \hline 0 \\ & 1 Levantamento ultradetalhado \\ & 2 Levantamento detalhado \\ & 3 Levantamento semidetalhado \\ & 4 Levantamento de reconhecimento detalhado \\ & 5 Levantamento de reconhecimento \\ & 6 Levantamento reconhecimento de baixa intensidade \\ & 7 Levantamento reconhecimento de média intensidade \\ & 8 Levantamento reconhecimento de alta intensidade \\ & 9 Levantamento exploratório-reconhecimento \\ & 10 Levantamento exploratório \\ & 11 Levantamento esquemático \\ & 12 Levantamento/mapa compilado \\ & \hline\end{aligned}

\section{Quadro 3. Tabela de domínio referente ao tipo de horizonte superficial}

\begin{tabular}{cl}
\hline Código & \multicolumn{1}{c}{ Descrição } \\
\hline 0 & Horizonte A fraco \\
1 & Horizonte A moderado \\
2 & Horizonte A chernozêmico \\
3 & Horizonte A proeminente \\
4 & Horizonte A antrópico \\
5 & Horizonte A húmico \\
6 & Horizonte A indiscriminado \\
7 & Horizonte turfoso \\
8 & \\
\hline
\end{tabular}

restrições que asseguram o ingresso dos dados conforme os domínios de valores preestabelecidos, diminuindo a possibilidade de entrada de dados inconsistentes e, ou, errados.

Nas figuras 4 a 6, são apresentados exemplos de telas, nas quais estão evidenciadas as interfaces gráficas do sistema, baseadas em caixas de texto para a entrada de dados numéricos, cadeias de caracteres, datas, textos longos e caixas de combinação para a entrada de dados de domínios definidos. Pode-se observar quea navegaçãoéà base de botões que abrem telas para outras entidades. Estes tipos de telas aproveitam el ementos visuais, pelo emprego de diferentes el ementos gráficos, nos quais intuitivamente o usuário, a partir do mouse ou do teclado, pode armazenar, atualizar ou excluir dados.

O SigSolos foi desenvolvido para ser capaz de armazenar dados que podem ser manuseados dentro de um enfoque de flexibilidade. Dessa forma, foram elaborados dois grupos de saídas, um de relatórios descritivos e outro de planilhas no formato Microsoft Excel. A opção pela planilha se deve ao fato de que, a partir delas, o usuário tem a possibilidadedetratar os dados em um grande el enco de aplicativos. 


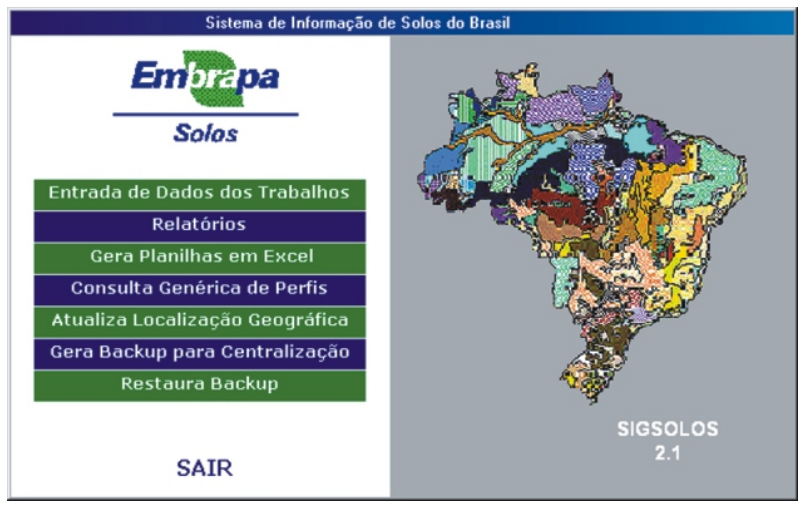

Figura 4. Tela de abertura.

(a) Trabalho

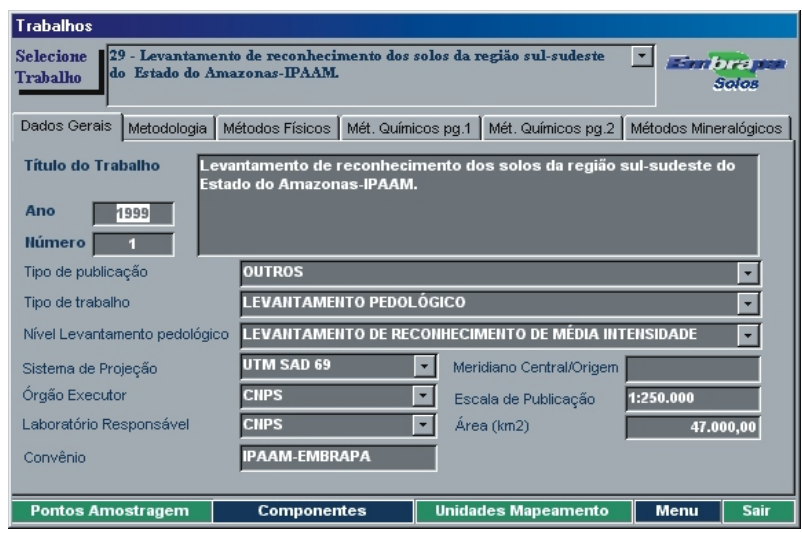

(b) Unidade de mapeamento

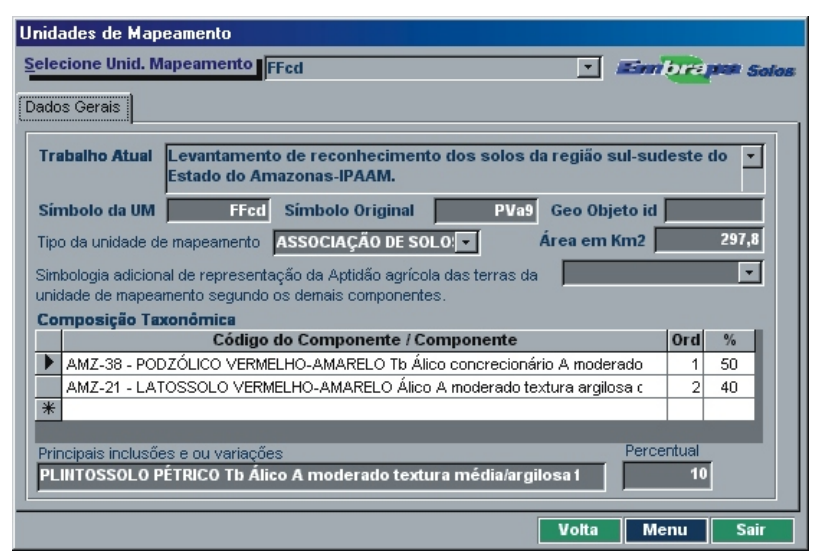

Figura 5. Tela principal da entidade trabalho.

Em 1998, foi concluída a 1aa etapa do SigSolos com a disponibilização da versão 1.0, no âmbito da Embrapa Solos (E mbrapa, 1998). Nos anos subseqüentes, esforços foram feitos no processo de entrada de dados alfanuméricos, ainda no âmbito da E mbrapa Solos, que culminou com o cadastramento de 75 levantamentos de solos, sendo a maioria executada pela Embrapa Solos e seus antecessores (a) Horizonte

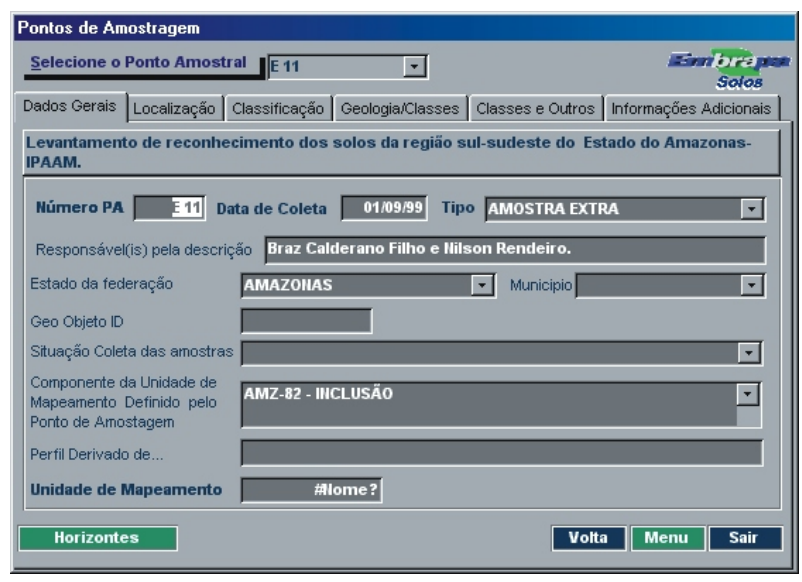

(b) Ponto de amostragem

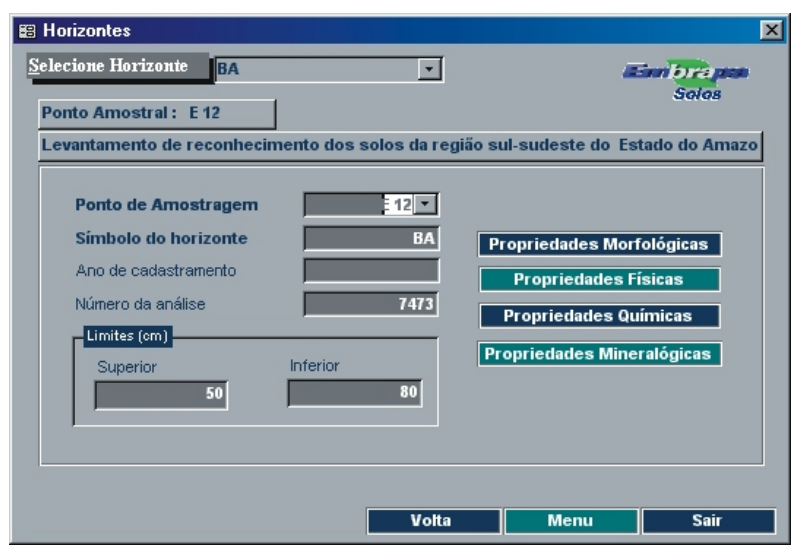

Figura 6. Tela principal da entidade unidade de mapeamento.

(SNLCS, por exemplo). Atualmente, a partir da I niciativa Sol os.br (www.cnps.embrapa.br/solosbr/), uma ação estratégica da Embrapa Solos para disponi bilização e aquisição contínua de dados sobre os solos brasileiros, já é possível acessar informações sobre estes levantamentos diretamente na internet.

Todo sistema de informações baseado em banco de dados, ao ser projetado, pressupõe um conjunto conhecido de aplicações. A pós sua instalação, via deregra, a disponibilidade dos dados e a capacidade de sua manipulação pelo SGBD despertam novas demandas não previstas na fase de projeto. No caso do SigSolos, por se tratar de um banco de dados associado a um SIG, as novas demandas deverão ser volumosas, seja por parte de pesquisadores da Ciência do Solo, seja por parte de outros usuários (Tanaka et al., 1997).

Segundo Petersen et al. (1999), uma descrição completa das apli cações de bancos de dados de sol os em todas as áreas das ciências ambientais é difícil de ser determinada. Enfatizando sua utilização no planejamento e manejo agrícola e atividades correlatas, como hidrologia e avaliação da qual idade 
da água, podem-se listar algumas aplicações fundamentais, tais como: estudos de planejamento de uso da terra, estudos de manejo de nutrientes e planejamento conservacionista, avaliação de recursos ambientais e modelagem, avaliação e planejamento de recursos regionais e gl obais.

Além das aplicações apresentadas anteriormente, aquelas relacionadas com a parametrização do Sistema Brasileiro de Classificação de Solos (ferramenta deauxílioà metodologia declassificação); com a geração de mapas de solos (a partir da reorganização de dados preexistentes) e de aptidão agrícola, aplicações geoestatísticas (Burrough, 1991) também poderão ser potencializadas pelo SigSolos. Da mesma forma, os dados armazenados no sistema servirão como fonte de informações para aplicações em sistemas especialistas (E mbrapa, 1995).

Vários sistemas de informações de solos foram desenvolvidos, nos últimos anos, para criar banco de dados de solo e tornar disponíveis aos usuários dados espaciais para diversas aplicações. Os si stemas de informações de sol os integram bancos de dados e ferramentas de SIG para analisar, recuperar, registrar, relatar e mostrar relacionamentos entre dados. Estes sistemas utilizam aplicativos de desenho de banco de dados comerciais, como INFORMIX eORACLE, ajudados por aplicativos de linguagem de programação (Petersen, et al., 1999).
No quadro 4, sãoapresentadas al gumas características dos princi pais sistemas de informações de sol os do mundo. Pode-se observar que o SigSol os guarda muitas semelhanças com estes sistemas, principal mente com o sistema canadense (CANSI S) e americano (NASIS). Neste sentido, destacam-se: o caráter espacial detodos os sistemas, característica desejável para os estudos que envolvem análise espacial; o tratamento dos dados alfanuméricos, através de um banco de dados comerciais (exceto o SOTER); o armazenamento de dados basicamente de instituições oficiais, e a utilização predominante de dados de levantamentos de solos.

Por outro lado, considerando as características das informações de sol os no Brasil eos recursos quase sempre escassos, optou-se pel o desenvolvimento de uma base única, não-escalar, que pudessearmazenar e tratar todo e qual quer tipo de informação gerada no País. Acrescenta-se a este fato a natureza ainda incipiente dos levantamentos de solos no que se refere de detalhe. Assim, noSigSol os convivem lado a lado, sem uma organização predeterminada, levantamentos generalizados (a maioria) e alguns poucos estudos semidetal hados e detal hados.

Outro aspecto a ser destacado é a escolha pela independência em relação ao aplicativo deSI G a ser utilizado. Outros sistemas, por estarem mais desenvolvidos do que oSigSol os, estabeleceram seus

Quadro 4. Características dos principais sistemas de informações de solos do mundo

\begin{tabular}{|c|c|c|c|c|}
\hline \multirow{2}{*}{ Característica } & \multicolumn{4}{|c|}{ Sistema de informação de solo } \\
\hline & SigSolos & Cansis $(1)$ & Nasis(2) & Soter (3) \\
\hline Caráter espacial & Sim & $\operatorname{Sim}$ & Sim & Sim \\
\hline Abrangência geográfica & Nacional/regional & $\begin{array}{l}\text { Nacional/regional/ } \\
\text { local }\end{array}$ & $\begin{array}{l}\text { Nacional/regional/ } \\
\text { local }\end{array}$ & Nacional \\
\hline Abrangência temática & Restrito & Restrito & Restrito & Restrito \\
\hline Aplicativo de SIG & Independente & ARC/INFO & ARC/INFO & ILWIS \\
\hline Modelo alfanumérico & Relacional & Relacional & Relacional & Tabular \\
\hline Fonte de dados & $\begin{array}{l}\text { Levantamentos } \\
\text { pedológicos e outros } \\
\text { estudos }\end{array}$ & $\begin{array}{l}\text { Levantamentos } \\
\text { pedológicos }\end{array}$ & $\begin{array}{l}\text { Levantamentos } \\
\text { pedológicos }\end{array}$ & $\begin{array}{l}\text { Levantamentos } \\
\text { Pedológicos }\end{array}$ \\
\hline $\begin{array}{l}\text { Procedimentos } \\
\text { Sistêmicos }\end{array}$ & Sim & Sim & Sim & Não \\
\hline Base Interinstitucional & Sim & Sim & Sim & Não \\
\hline $\begin{array}{l}\text { Imagens Sensores } \\
\text { Remotos }\end{array}$ & Não & Não & Não & Não \\
\hline Base Ú nica - não-escalar & Sim & Não & Não & Sim \\
\hline Redução da informação & $\operatorname{Sim}$ & Sim & Não & Sim \\
\hline Dados absolutos & Sim & Sim & Sim & Não \\
\hline Dados interpretativos & Não & Sim & Sim & Não \\
\hline Origem dos dados & Instituições oficiais & Instituições oficiais & Instituições oficiais & Instituições oficiais \\
\hline Operações principais & $\begin{array}{l}\text { Classificação, } \\
\text { Generalização e } \\
\text { Análises }\end{array}$ & $\begin{array}{l}\text { Classificação, } \\
\text { Generalização e } \\
\text { Análises }\end{array}$ & $\begin{array}{l}\text { Classificação, } \\
\text { Generalização e } \\
\text { Análises }\end{array}$ & $\begin{array}{l}\text { Classificação e } \\
\text { Generalização }\end{array}$ \\
\hline
\end{tabular}

(1) CANSIS - Canadian Soil Information System. (2) NASIS - National Soil Information System. ${ }^{(3)}$ SOTER - The World Soil and Terrain Database. 
aplicativos de SIG com base nas características de suas informações e, principalmente, no desempenho destes. Porém, hoje já se tem certeza de que a utilização do Arcl nfo (ESRI, 1994) éa mel hor opção dentre as disponíveis no mercado, dada a confiabilidade e robustez deste aplicativo.

\section{PERSPECTIVAS FUTURAS}

Durante o desenvolvimento do SigSolos, muito esforço foi dispensado na busca da organização das informações de sol os do Brasil, principal mente, pel o fato de estarem essas informações muito dispersas e pouco normatizadas. Este trabal ho culminou com a liberação de uma versão inicial capaz de, nesta primeira etapa, atender às necessidades iniciais do projeto. Entretanto, al guns pontos carecem demaior atenção, para que seja alcançado um sistema, a exemplo do americano e do canadense, capaz de gerenciar o vasto e vali ioso vol ume de dados de sol os, tão importante para a sustentabilidade de nossos ecossistemas.

Nestesentido, os responsáveis pelo desenvolvimento doSigSolos devem dar atenção aos seguintes pontos: (a) mi gração dos dados do Microsoft Access para um banco de dados relacional robusto; (b) definição oficial do software de SI G a ser utilizado; (c) atualização dos dados, com base noSistema Brasileiro de Classificação de Solos (E mbrapa, 1999); (d) disponi bilização da base de dados em universidades e outras instituições estaduais e federais; e (e) digitalização dos mapas de solos gerados nas diferentes instituições oficiais, com base em um critério único, com vistas em obter maior uniformização e confiabilidade dos produtos gerados.

Dados temáticos correlatos, como geologia e geomorfologia, e informações de natureza sócioeconômica devem ser incorporados ao sistema, por meio da elaboração de convênios com instituições oficiais, de forma a viabilizar o estabel ecimento de um sistema cooperativo, em que cada instituição seria responsável por um conjunto restrito de temas de sua competência. A estrutura do sistema deve ser calcada em procedimentos, em equipamentos e em aplicativos consagrados, para que possa servir de base e de testemunho ao desenvolvimento tecnológico nos processos de captura e manipulação de dados ambientais, bem como ser capaz de atender a requisitos de interpretações.

\section{CONCLUSÕES}

1. O banco de dados e a visualização geográfica através de SIG podem ser gerados em qualquer software utilizado no mercado.
2. Os dados alfanuméricos adquiridos pelo subsistema de entrada de dados em MS Access podem ser importados pelo SGBD adotado.

3. Dadas as suas características, o SigSolos pode contribuir para maior organização e sistematização da informação de sol os no Brasil.

4. O processo de automatização do programa oferece aos usuários das informações de sol os acesso rápido para armazenamento, atual ização e exclusão de dados; ligação, em tempo real, entre mapas e dados al fanuméricos relacionados com estes mapas, edesenvolvimento de novas funcionalidades, a saber: sistemas de avaliação agrícola, sistemas especialistas de suporte à decisão e de elaboração de zoneamentos.

\section{LITERATURA CITADA}

BAUMGARDNER, M.F. Soil databases. In: SUMNER, M.E., ed. Handbook of soil science. Boca Raton, CRC Press, 1999. p.H1-H4.

BURROUGH, P.A. Soil information systems. In: MAGUIRE, D.J .; GOODCHILD, M.F. \& RHIND, D.W., ed. Geographical information systems: principles and applications. Essex, Longman Scientific and Technical, 1991. v.2. p.153-169.

CHEN, P.P. The entity-relationship model - toward a unified view of data. ACM Transactions on Database Systems, 1:936, 1976.

COMPUTER ASSOCIATION. OpenIngres: SQL Language Reference. New York, 1995.

COOTE, D.R. \& MACDONALD, K.B. The canadian soil database. In: SUMNER, M.E., ed. Handbook of soil science. Boca Raton, CRC Press, 1999. p.H41-H51.

CURI, N.; LARACH, J .O.I.; KAMPF, N.; MONIZ, A.C. \& FONTES, L.E.F. Vocabulário de ciência do solo. Campinas, Sociedade Brasileira de Ciência do Solo, 1993. 90p.

EMPRESA BRASILEIRA DE PESQUISA AGROPECUÁRIA EMBRAPA. Centro Nacional de Pesquisa de Solos (Rio de J aneiro, RJ ). Base de informações georreferenciada de sol os: metodol ogia e guia bási co do aplicativo SigSol os, versão 1.0. Rio deJ aneiro, 1998. CD-ROM (Embrapa - CNPS. Boletim de pesquisa, 11)

EMPRESA BRASILEIRA DE PESQUISA AGROPECUÁRIA EMBRAPA. Centro Nacional de Pesquisa de Solos (Rio de J aneiro, RJ ). Iniciativa Solos.br. Disponível em: < http:// www.cnps.embrapa.br/solosbr > acesso em: 26 fev. 2003.

EMPRESA BRASILEIRA DE PESQUISA AGROPECUÁRIA EMBRAPA. Centro Nacional de Pesquisa de Solos (Rio de J aneiro, RJ ). Manual de métodos de análise de solos. 2.ed. Rio de J aneiro, 1997. 212p. (Embrapa - CNPS. Documentos, 1)

EMPRESA BRASILEIRA DE PESQUISA AGROPECUÁRIA EMBRAPA. Centro Nacional de Pesquisa de Solos (Rio de J aneiro, RJ ). Procedimentos normativos de levantamentos pedológicos. Brasília, 1995. 116p. 
EMPRESA BRASILEIRA DE PESQUISA AGROPECUÁRIA EMBRAPA. Centro Nacional de Pesquisa de Solos (Rio de J aneiro, RJ ). Sistema Brasileiro de Classificação de Solos. Brasília, Embrapa Produção da I nformação, 1999. 412p.

EMPRESA BRASILEIRA DE PESQUISA AGROPECUÁRIA EMBRAPA. Serviço Nacional de Levantamento e Conservação de Solos (Rio de J aneiro, RJ ). Critérios para distinção de classes de solos e de fases de unidades de mapeamento: normas usadas pel o SNLCS. Rio de J aneiro, 1988a. 67p. (E mbrapa - SNLCS. Documentos, 11)

EMPRESA BRASILEIRA DE PESQUISA AGROPECUÁRIA EMBRAPA. Serviço Nacional de Levantamento e Conservação de Solos (Rio de J aneiro, RJ ). Definição e notação de horizontes e camadas do solo. Rio de J aneiro, 1988b. 54p. (Embrapa - SNLCS. Documentos, 3)

ENVIRONMENTAL SYSTEMS RESEARCH INSTITUTE ESRI. ArcView GIS: The Geographic I nformation System for everyone. New York, 1996.

ENVIRONMENTAL SYSTEMS RESEARCH INSTITUTE ESRI. PC Arc/l nfo: Command references. New York, 1994.

ERNSTROM, D.J . \& LYTLE, D.J . Enhanced soils information systems from advances in computer technol ogy. Geoderma, 60:327-341, 1993.

FAO. The digitized soil map of the world including derived soil properties. Rome, FAO, 1996. CD-ROM.

FERNÁNDEZ, R.N. \& RUSINKIEWICZ, M. A conceptual design of a soil database for a geographic information system. Int. J . Geogr. Inf. System, 7:525-539, 1993.

LEMOS, R.C. \& SANTOS, R.D. Manual de descrição e col eta de solo no campo. 3.ed. Campinas, Sociedade Brasileira de Ciência do Solo, 1996. 84p.

LYTLE, D.J . Digital soils databases for the United States. In: Environmental modeling with GIS. New York, Oxford University Press, 1993. p.386-391.

LYTLE, D.J . United States soil survey database. In: SUMNER, M.E., ed. Handbook of soil science. Boca Raton, CRC Press, 1999. p.H53-H67.
MACDONALD, K.B. \& KLOOSTERMAN, B. The canadian soil information system - CANSIS: general users manual. Ottawa, Land Resource Research Institute, Research Branch, Agriculture Canada, 1984. 56p.

NACHTERGAELE, F.O. From the soil map of the world to the digital global soils and terrain database: 1960 - 2002. In: SUMNER, M.E., ed. Handbook of soil science. Boca Raton, CRC Press, 1999. p.H5-H17.

PETERSEN, G.W.; NIZEYIMANA, E.; MILLER, D.A. \& EVANS, B.M. The use of soil databases in resource assessments and land use planning. In: SUMNER, M.E., ed. Handbook of soil science. Boca Raton, CRC Press, 1999. p.H69-H94.

RAMALHO FILHO, A. \& BEEK, K.J. Sistema de avaliação da aptidão agrícola das terras. 3. ed. rev. Rio de J aneiro, Empresa Brasileira de Pesquisa Agropecuária, 1995. 65p.

SOIL SURVEY STAFF - SSS. National soil information system (NASIS): soil interpretation and information dissemination sub-system. Draft requirements statement. Lincoln, USDA, Natural Resources Conservation Service, National Soil Survey Center, 1991. 67p.

SOIL SURVEY STAFF - SSS. Soil taxonomy: a basic system of soil classification for making and interpreting soil surveys. Washington, USDA, Natural Resources Conservation Service, 1999. 870p. (Agricultural Handbook, 436)

TANAKA, A.K.; BHERING, S.B. \& CHAGAS, C.S. Modelagem conceitual do sistema de informações georreferenciadas de solos do Brasil. In: SOLO SUELO - CONGRESSO LATINOAMERICANO DE CIÊNCIA DO SOLO, 13., Águas de Lindóia, 1996. Resumos expandidos. Águas de Lindóia, Sociedade Brasileira de Ciência do Solo, 1996. CD-ROM.

TANAKA, A.K.; CHAGAS C. S. \& BHERING, S.B. O sistema de informaç̃̃es georreferenciadas de solos do Brasil. In: CONGRESSO BRASILEIRO DE CIÊNCIA DO SOLO, 26. Rio deJ aneiro, 1997. Resumos expandidos. Rio deJ aneiro, Sociedade Brasileira de Ciência do Solo, 1997. CD-ROM.

van ENGELEN, V.W.P. \& WEN, T.T. Global and national soils and terrain digital databases (SOTER): procedures manual. Wageningen, UNEP-ISSS-ISRIC-FAO, 1995. 129p.

van ENGELEN, V.W.P. SOTER: the world soils and terrain database. In: SUMNER, M.E., ed. Handbook of soil science. Boca Raton, CRC Press, 1999. p.H19-H28. 